\title{
ORBIT-CLOSURE DECOMPOSITIONS AND ALMOST PERIODIC PROPERTIES
}

\author{
w. H. GOTTSCHALK
}

Let $X$ be a metric space with metric $\rho$, let $f(X) \subset X$ be a continuous mapping, and let $h(X)=X$ be a homeomorphism. For $x \in X$, the set $\sum_{n=0}^{+\infty} f^{n}(x)$ is called the semi-orbit of $x$ under $f$ and the set $n \sum_{-\infty}^{+\infty} h^{n}(x)$ is called the orbit of $x$ under $h$. For $x \in X$, the closure of the semi-orbit of $x$ under $f$ is called the semi-orbit-closure of $x$ under $f$ and the closure of the orbit of $x$ under $h$ is called the orbit-closure of $x$ under $h$.

A nonvacuous subset $Y$ of $X$ is said to be semi-minimal (minimal) under $f(h)$ provided that the semi-orbit-closure (orbit-closure) of each point of $Y$ is $Y$. Clearly, any two semi-minimal (minimal) sets are either coincident or disjoint. It is easily proved that a subset $Y$ of $X$ is semi-minimal (minimal) under $f(h)$ if and only if $Y$ is nonvacuous, closed, $f(Y) \subset Y(h(Y)=Y)$, and furthermore $Y$ contains no proper subset with these properties. We follow Birkhoff $[2, \text { p. 198 }]^{1}$ in the terminology of "minimal set."

A decomposition of $X$ is defined to be a collection of nonvacuous closed pairwise disjoint subsets of $X$ which fill up $X$. We say that the mapping $f$ gives a semi-orbit-closure (a semi-minimal set) decomposition provided that the collection of semi-orbit-closures (semi-minimal sets) is a decomposition of $X$. Also, it is said that the homeomorphism $h$ gives an orbit-closure (a minimal-set) decomposition provided that the collection of orbit-closures (minimal sets) is a decomposition of $X$.

A point $x$ of $X$ is said to be almost periodic under $f$ provided that to each $\epsilon>0$ there corresponds a positive integer $N$ with the property that in every set of $N$ consecutive positive integers appears an integer $n$ such that $\rho\left(x, f^{n}(x)\right)<\epsilon$. The mapping $f$ is said to be pointwise almost periodic provided that each point of $X$ is almost periodic under $f$. It is to be noted that various writers use the above terms in different senses and employ other terminologies for these notions.

LEMMA 1. The mapping $f$ (homeomorphism $h$ ) gives a semi-orbitclosure (an orbit-closure) decomposition if and only if $f(h)$ gives a semiminimal-set (a minimal-set) decomposition; and in either event, the two decompositions coincide.

Presented to the Society, August 14, 1944 ; received by the editors May 15, 1944 and November 15, 1944.

1 Numbers in brackets refer to the bibliography at the end of the paper. 
The proof is easy and will be omitted.

LeMma 2. In order that the homeomorphism $h$ give an orbit-closure decomposition it is sufficient that $h$ give a semi-orbit-closure decomposition; and in case $X$ is compact, this condition is also necessary. In either event, the two decompositions coincide.

Proof. The proof of the sufficiency is easy and will be omitted. We establish the necessity. Let $C$ be an orbit-closure. By Lemma 1, it is enough to show that $C$ is a semi-minimal set. Let $Y$ be a nonvacuous closed subset of $C$ such that $h(Y) \subset Y$. The proof will be completed if we show $Y=C$. Define $Z=\prod_{n=0}^{+\infty} h^{n}(Y)$. Now $Z$ is a nonvacuous closed subset of $C$ such that $h(Z)=Z$. Since $C$ is a minimal set by Lemma $1, Z=C$ and, hence, $Y=C$.

LEMma 3. If $x \in X$ is almost periodic under $f$, then the semi-orbitclosure $C$ of $x$ is semi-minimal.

Proof. Suppose $C$ is not semi-minimal. Then there exists a point $y$ of $C$ such that the semi-orbit-closure $Y$ of $y$ is not $C$. Now $Y \subset C$. Also $x \notin Y$, since otherwise $C \subset Y$ and thus $Y=C$. Let $2 \epsilon$ be the distance from $x$ to $Y$. There exists a positive integer $N$ such that in every set of $N$ consecutive positive integers appears an integer $n$ so that $\rho\left(x, f^{n}(x)\right)<\epsilon$. Choose $\delta>0$ so small that $z \in X$ with $\rho(y, z)<\delta$ implies $\rho\left(f^{i}(y), f^{i}(z)\right)<\epsilon(i=1,2, \cdots, N)$. Now there exists a nonnegative integer $p$ such that $\rho\left(y, f^{p}(x)\right)<\delta$. Also it is possible to find an integer $q, 1 \leqq q \leqq N$, so that $\rho\left(x, f^{p+q}(x)\right)<\epsilon$. Hence, $\rho\left(x, f^{q}(y)\right)<2 \epsilon$ which is impossible.

Lemma 4. If $X$ is locally compact and if the subset $Y$ of $X$ is semiminimal under $f$, then each point of $Y$ is almost periodic.

Proof. Assume some point $x$ of $Y$ is not almost periodic. There exists a neighborhood $U$ of $x$ such that $\bar{U}$ is compact and such that for each positive integer $n$ there exists a point $x_{n}$ of $U \cdot Y$ with the property that $f^{m}\left(x_{n}\right) \notin U(m=1,2, \cdots, n)$. Some subsequence of $x_{1}, x_{2}, \cdots$ converges to some point, say $y$, of $\bar{U} \cdot Y$. There exists a positive integer $M$ such that $f^{M}(y) \in U$ and, hence, there also exists a neighborhood $V$ of $y$ such that $f^{M}(V) \subset U$. Choose an integer $N$ so that $N>M$ and $x_{N} \in V$. Then, $f^{M}\left(x_{N}\right) \in U$ which is a contradiction.

THEOREM 1. In order that the mapping $f$ give a semi-orbit-closure decomposition, it is sufficient that $f$ be pointwise almost periodic; and in case $X$ is locally compact, this condition is also necessary.

The proof follows easily from Lemmas 1, 3 and 4 . 
THEOREM 2. In order that the homeomorphism $h$ give an orbit-closure decomposition it is sufficient that $h$ be pointwise almost periodic; and in case $X$ is compact, this condition is also necessary.

The proof proceeds easily from Theorem 1 and Lemma 2.

The preceding results are related to certain results of Birkhoff [2, p. 199] and Hall and Kelley [4]. Gottschalk [3] contains a direct proof of Theorem 2.

We pause in our main development to comment on the rôle of local compactness in the second part of Theorem 1 . In this case the semiorbit-closures are actually compact, as the following indicates.

TheOREM A. If $x \in X$ is almost periodic under $f$ and if there exists a neighborhood $U$ of $x$ whose closure is compact, then the semi-orbitclosure of $x$ is itself compact.

Proof. There exists an integer $N$ and a sequence $\left\{n_{i} \mid i=0,1, \cdots\right\}$ of integers such that $0=n_{0}<n_{1}<\cdots, n_{i+1}-n_{i} \leqq N$ and $f^{n_{i}}(x) \in U$ for all $i$. Define $K=\sum_{r=0}^{N} f^{r}(\bar{U})$. Now $K$ is compact. We show the semi-orbit of $x$ is contained in $K$, which completes the proof. Let $n$ be any non-negative integer. There exists a non-negative integer $i$ such that $n_{i} \leqq n \leqq n_{i+1}$. Hence, $f^{n}(x)=f^{n-n_{i}} f^{n_{i}}(x) \in f^{n-n_{i}}(U) \subset K$.

THEOREM B. If $Y \subset X$ is semi-minimal under $f$ and if $Y$ intersects a neighborhood $U$ whose closure is compact (in particular, if $X$ is locally compact), then $Y$ is itself compact.

Proof. Let $y \in Y \cdot U$. By the argument used in the proof of Lemma 4 , it can be shown that $y$ is almost periodic. The conclusion now follows from Theorem A. (Theorem B is not valid for a minimal set as the example of a discrete infinite orbit shows.)

Besicovitch [1] has constructed an interesting example of a homeomorphism of the plane onto itself which possesses some semiorbits dense in the plane and which leaves the origin fixed. He seems to remark at the end of his paper (p. 65) that every semi-orbit, excluding the origin, is also dense in the plane. Theorem B would indicate that either this remark or our interpretation of it is in error. For, take $X=Y$ to be the plane with the origin deleted. If every semi-orbit is dense in the punctured plane, then the punctured plane would be compact. Question: In Besicovitch's example [1], is the orbit of every point of the plane, excepting the origin, dense in the plane? We now continue with the main sequence of theorems.

Let $\left\{X_{n} \mid n=1,2, \cdots\right\}$ be a sequence of subsets of $X$. The set of all points $x$ of $X$ such that each neighborhood of $x$ intersects $X_{n}$ for 
almost all (infinitely many) positive integers $n$ is denoted by $\lim \inf \left\{X_{n}\right\}$ (lim sup $\left.\left\{X_{n}\right\}\right)$. In case $\lim \inf \left\{X_{n}\right\}=\lim \sup \left\{X_{n}\right\}$, we denote this set by $\lim \left\{X_{n}\right\}$. Of course, for any sequence $\left\{X_{n}\right\}$, $\lim \inf \left\{X_{n}\right\} \subset \lim \sup \left\{X_{n}\right\}$.

Let $D$ be a decomposition of $X$. For $x \in X$, let $D(x)$ denote the element of $D$ containing $x$. The decomposition $D$ is said to be continuous provided that $x_{0}, x_{n} \in X(n=1,2, \cdots)$ with $x_{n} \rightarrow x_{0}$ implies $D\left(x_{0}\right)$ $C \lim \inf \left\{D\left(x_{n}\right)\right\} \subset \lim \sup \left\{D\left(x_{n}\right)\right\} \subset D\left(x_{0}\right)$.

Following G. A. Hedlund, we say the mapping $f$ is uniformly pointwise almost periodic provided that to each $\epsilon>0$ there corresponds a positive integer $N$ such that if $x \in X$, then in every set of $N$ consecutive positive integers appears an integer $n$ so that $\rho\left(x, f^{n}(x)\right)<\epsilon$. Clearly, if $f$ is uniformly pointwise almost periodic, then $f$ is pointwise almost periodic.

Lemma 5. For $x \in X$, let $C(x)$ denote the semi-orbit-closure of $x$ under $f$. If $x_{0}, x_{n} \in X(n=1,2, \cdots)$ with $x_{n} \rightarrow x_{0}$, then $C\left(x_{0}\right) \subset \lim$ inf $\left\{C\left(x_{n}\right)\right\}$.

Proof. Let $x \in C\left(x_{0}\right)$ and let $U$ be any neighborhood of $x$. For some non-negative integer $k, f^{k}\left(x_{0}\right) \in U$. By the continuity of $f^{k}, f^{k}\left(x_{n}\right) \in U$ for almost all positive integers $n$, that is, $U$ intersects $C\left(x_{n}\right)$ for almost all positive integers $n$. The conclusion follows.

THEOREM 3. In order that the mapping $f$ give a continuous semi-orbitclosure decomposition it is sufficient that $f$ be uniformly pointwise almost periodic; and in case $X$ is compact, this condition is also necessary.

Proof. We establish the sufficiency. Let $D$ denote the collection of semi-orbit-closures. By Theorem $1, D$ is a decomposition of $X$. By Lemma 5, it is enough to prove that lim sup $\left\{C\left(x_{n}\right)\right\} \subset C\left(x_{0}\right)$ for $x_{0}$, $x_{n} \in X(n=1,2, \cdots)$ with $x_{n} \rightarrow x_{0}$, where $C(x)$ denotes the semi-orbitclosure of the point $x$. Assume this is false. Then there exist points $x_{0}, x_{n} \in X(n=1,2, \cdots)$ such that $x_{n} \rightarrow x_{0}$ and $\lim \sup \left\{C\left(x_{n}\right)\right\}$

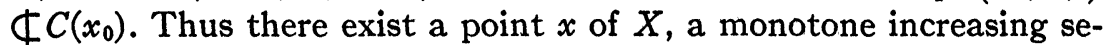
quence $n_{1}, n_{2}, \cdots$ of positive integers, and a sequence $m_{1}, m_{2}, \cdots$ of non-negative integers such that $f^{m_{i}}\left(x_{n_{i}}\right) \rightarrow x$ and $x \in C\left(x_{0}\right)$. Hence, $C(x) \cdot C\left(x_{0}\right)=\Lambda$ and $x_{0} \notin C(x)$. Let $2 \epsilon$ denote the distance from $x_{0}$ to $C(x)$. Since $f$ is uniformly pointwise almost periodic, there exists a positive integer $k$ such that for each positive integer $i$ it is possible to find an integer $k_{i}$ with the properties that $1 \leqq k_{i} \leqq k$ and $\rho\left(x_{n_{i}}, f^{m_{i}+k_{i}}\left(x_{n_{i}}\right)\right)<\epsilon$. There exists an integer $k_{0}$ such that $k_{i}=k_{0}$ for infinitely many positive integers $i$. Since also $x_{n_{i}} \rightarrow x_{0}$ and $f^{m_{i}+k_{0}}\left(x_{n_{i}}\right)$ $\rightarrow f^{k_{0}}(x)$, we have $\rho\left(x_{0}, f^{k_{0}}(x)\right) \leqq \epsilon<2 \epsilon$. Hence, the distance from $x_{0}$ to $C(x)$ is less than $2 \epsilon$. This is a contradiction. 
We establish the necessity. Suppose $f$ is not uniformly pointwise almost periodic. Then there exists a positive number $\epsilon$ such that for each positive integer $n$ it is possible to find a positive integer $m_{n}$ and a point $x_{n}$ of $X$ such that $N_{c}\left(x_{n}\right) \cdot \sum_{i=0}^{n} f^{m_{n}+i}\left(x_{n}\right)=\Lambda$, where $N_{\delta}(y)$ denotes the $\delta$-neighborhood of the point $y$. We may suppose that $x_{n} \rightarrow x_{0}$ and $f^{m_{n}}\left(x_{n}\right) \rightarrow x$ for some points $x_{0}, x$ of $X$. For all sufficiently large positive integers $n, N_{\epsilon / 2}\left(x_{0}\right) \cdot \sum_{i=0}^{n} f^{m_{n}+i}\left(x_{n}\right)=\Lambda$. It follows that $N_{\epsilon / 2}\left(x_{0}\right) \cdot C(x)=\Lambda$ and $x_{0} \notin C(x)$, where $C(y)$ denotes the semiorbit-closure of the point $y$. Now $\lim \left\{C\left(x_{n}\right)\right\}=C\left(x_{0}\right)$ and $C(x)$ $=\lim \left\{C\left(f^{m_{n}}\left(x_{n}\right)\right)\right\}=\lim \left\{C\left(x_{n}\right)\right\}$. Hence, $C\left(x_{0}\right)=C(x)$ and $x_{0} \in C(x)$. This is a contradiction.

THEOREM 4. In order that the homeomorphism $h$ give a continuous orbit-closure decomposition it is sufficient that h be uniformly pointwise almost periodic; and in case $X$ is compact, this condition is also necessary.

The proof follows readily from Theorem 3 and Lemma 2.

It is worthy of note that if $X$ is compact, then there exists a subset $Y$ of $X$ such that $f(Y)=Y$ is uniformly pointwise almost periodic. The proof is short. The property $\mathrm{P}$ of being a nonvacuous closed subset $Z$ of $X$ such that $f(Z) \subset Z$ is easily shown to be inducible. By the Brouwer reduction theorem, there exists a subset $Y$ which has property $\mathrm{P}$ irreducibly. Then $Y$ is semi-minimal and $f(Y)=Y$, since $f(Y)$ has property $\mathrm{P}$. The conclusion now follows from Theorem 3.

\section{BIBLIOGRAPHY}

1. A. S. Besicovitch, $A$ problem on topological transformation of the plane, Fund. Math. vol. 28 (1937) pp. 61-65.

2. G. D. Birkhoff, Dynamical systems, Amer. Math. Soc. Colloquium Publications, vol. 9, New York, 1927.

3. W. H. Gottschalk, Powers of homeomorphisms with almost periodic properties, Bull. Amer. Math. Soc. vol. 50 (1944) pp. 222-227.

4. D. W. Hall and J. L. Kelley, Periodic types of transformations, Duke Math. J. vol. 8 (1941) pp. 625-630.

UNIVERSITY OF VIRGINIA AND UNIVERSITY OF RICHMOND 